\title{
Emerging Noninvasive Neurostimulation Technologies: CN-NINM and SYMPATOCORECTION
}

\author{
Yuri P. Danilov'1, Vladimir S. Kublanov² \\ ${ }^{1}$ Tactile Communication and Neuromodulation Laboratory, Biomedical Engineering Department, University of \\ Wisconsin-Madison, 1550 Engineering Drive, Madison, USA \\ ${ }^{2}$ Research Medical and Biological Engineering Center of High Technologies, Institute of Radio Engineering and \\ Information Technology, Ural Federal University, Mira Str. 32, Ekaterinburg, Russia \\ Email: ydanilov@wisc.edu, kublanov@mail.ru
}

Received 8 January 2014; revised 12 February 2014; accepted 19 February 2014

Copyright () 2014 by authors and Scientific Research Publishing Inc.

This work is licensed under the Creative Commons Attribution International License (CC BY).

http://creativecommons.org/licenses/by/4.0/

(c) (1) Open Access

\section{Abstract}

Two emerging noninvasive neurostimulation technologies, based on electrocutaneius stimulation of the tongue (CN-NINM) and the neck (SYMPATOCORRECTION) are presented. Currently, two portable devices were developed and introduced in clinical practice: PoNS ${ }^{\mathrm{TM}}$ (portable neurostimulator) and SYMPATOCOR. Both technologies are complements of each other and demonstrate perspectives in various applications for purpose of neurorehabilitation and neurological symptoms management in such difficult rehabilitation areas, as traumatic brain injury, stroke, Parkinson's disease, multiple sclerosis and many other neurological disorders.

\section{Keywords}

\section{Neurostimulation; Neurorehabilitation; Noninvasive; Medical Device}

\section{Introduction}

It is difficult to find a more challenging problem in rehabilitation medicine than recovery (either full or partial) of neurological function that is lost due to injury, disease, or aging. There are many investigations on physiological and psychiatric brain dysfunctions.

There are also results of clinical applications of various drugs, devices, and methods for rehabilitation.

Nevertheless, this problem has grown particularly acute with increasing life spans and dramatically increasing survival periods after major diseases and traumas that lead to ever increasing numbers of people with substantial 
neurological dysfunctions.

The main objective of the current paper is to introduce two new noninvasive technologies to medical and biomedical engineering community, who is working in the field of neurostimulation and neuromodulation.

From anatomical and physiological point of view, both presented original technologies are complimentary to well-known deep brain (DBS) and vagal nerve (VNS) stimulation methods, which, however, are both invasive and approved by FDA.

\section{Neurorehabilitation Problems}

Most neurological disorders are chronic conditions which might cause severe physical cognitive and emotional disability. They affect not only the patients but also their families and caregivers, since these disorders require constant attention and treatment and have a huge impact to the proper functioning of an individual in society.

Neurological disorders represent ones of the central or peripheral nervous systems. Basically, they can be considered damaging the whole or part of the nervous system due to structural, biochemical, or electrical abnormalities. The disorders might imply a change in the circuitry of the neural pathways, which makes the treatment of these conditions much more difficult as the physical connections between neurons are no longer present or they are entirely changed.

Although an extensive search has been carried out in the field of neurology, many aspects of the inner operation of the central nervous system still remain unknown. Thus, scientists are encountering difficulties in providing efficient solutions in the treatment of neurological conditions.

So far, medication is one of the most commonly used methods in the treatment of neurological disorders. Due to achievements in neuropharmacology, numerous treatment options are given to patients to improve their overall lifestyle. However, the adverse effects of drugs are not to be neglected. Note that majority of drugs are prescribed systematically that affects onto the entire body of patients. Under this, the adverse effects can limit the patient's lifestyles. Furthermore, about $20 \%$ of patients are not responsive to the medication or become resistant to treatment over time along with progression of the disease [1].

In these cases, neuromodulation might be a viable solution. Neuromodulation is considered to be a new method of treatment for neurological disorders. It involves direct application of electrical, chemical, or biological stimulation of the affected region of the nervous system. In recent years, neuromodulation has proved to be a feasible alternative in the treatment of the increasingly common neurological conditions together with other advanced branches of neurotechnologies such as the stem cells therapy, tissue engineering, neural repair, and regeneration.

\section{Neurostimulation}

Neurostimulation technologies (as a part of more general field of neuromodulation) are oriented to physical activation of the neural tissue of the central nerve system (CNS) including brain, spinal cord, and peripheral nerves. Nowadays, the area of neurostimulation is developing by research activity across basic and clinical domains.

Currently, few dozen forms of neurostimulation are undergoing development and evaluation as interventions for neurological and psychiatric disorders including traditional use of electrical or magnetic stimulation, use of ultrasound, radio waves, and optical stimulation to alter excitability of neural tissue. Stimulation techniques are a unique form of treatment that principally differs from pharmacology, psychotherapy, or physical therapy.

The neurostimulation therapies range from noninvasive techniques such as transcranial magnetic stimulation (TMS), which applies single or repetitive stimuli at the scalp surface, to deep brain stimulation (DBS), which involves neurosurgical implantation of electrodes in specific brain regions, and up to the vagal nerve stimulation, which also involve implantation of electrodes to directly stimulate left vagal nerve. These interventions differ in many fundamental characteristics such as: whether stimulation results in seizures or is nonconvulsive, is continuous or intermittent, or use the brain activity to determine the timing or site of stimulation.

\subsection{Neurostimulation Types}

Few dozens of neurostimulation technologies were developed in past ten years, as a result of search for new effective methods of neurorehabilitation. There are many ways to sort out or classify different types of neurostimulation. 


\subsubsection{Body (Spinal Cord and Peripheral Nerves) vs. Brain}

Let's consider brain and body as two independent parts and suppose them to be different targets of stimulation and different areas of rehabilitation. Also assume that neurological disorders lead to malfunctioning areas and regions of the brain, spinal cord, peripheral nerve, and muscles. The body includes the spinal cord, peripheral nerves, and muscles. The cranial nerves are different from the spinal ones (including cervical nerves, level C1C4), the cranial nerves are directly linked with a brain, not with the spinal cord. The TMS and DBS are brain oriented stimulations, the ETS is a body oriented neurostimulation, the vagal nerve stimulation (VNS) is an example of cranial nerve activation.

\subsubsection{Invasive vs. Noninvasive Stimulation}

The difference is in methods of stimulation, namely, with or without breaking the skin surface.

Sensory (afferent) vs. motor (efferent) stimulation. Sensory stimulation oriented to intensive stimulation of sensory receptors (visual, audio, tactile, and etc.) to provide information into the brain (centripetally from the body to the brain). The motor stimulation is mainly oriented to centrifugal activation of the body, activation from the brain to the body by activation of muscles and/or nerves, and creation of motion (TESS, NESS neurostimulation systems).

\subsubsection{External Stimulation vs. Internal Stimulation}

Natural source of the brain activation are neural impulses or spikes that are generated by billions of neural specialized receptors located in depth of skin or internal body tissues. That is internal natural stimulation. Impulses are streaming to the spinal cord and brain by nerves (the nerve trunk consists of thousands nerve fibers). Further, the impulses are distributed across multiple brain structures and activate complex neuronal networks. This activation is performed by using naturally designed spatial and temporal patterns that are unique for different brain structures and are based on anatomical and physiological types of neurons and patterns of interneuron connections.

Artificial or other (not natural) activations of the brain structures by electrical or magnetic fields, electrical current, light, or ultrasound (usually applied from outside the body or scull) can be considered as external, and they fundamentally differ from natural (internal) activations.

\subsubsection{Global Stimulation vs. Local Stimulation}

All kinds of transcranialstimulation, namely, magnetic (TMS), electrical (TES), transcranial direct current stimulation (TDCS), alternating current (TACS), or random noise (TRNS) can be considered as a global. This is because initially the large volumes of the brain tissue are affected by such stimulation that spreads the corresponding activation in different cortical and subcortical areas of the CNS. In contrast, the deep brain stimulation (DBS) activates a small volume of the brain tissue mainly located in/or around a single subcortical structure (need a stereotaxic precision of electrode positioning), i.e., it is assumed to affect a particular part of the functional network [2].

By definition, the neurorehabilitation is oriented to recovery of missing/malfunctioning physiological, cognitive, or mental function as a result of some neural damage. Such a damage (a small CVA) can or cannot be localized (under a diffused TBI), can be located on many levels of the brain (brainstem, cerebellum, basal ganglia, and thalamus) or one or many cortical areas or can be a combination of all abovementioned sites. Small or large, single or multiple damages can produce significant distortion of one or many sensory, motor, or cognitive functions such as balance, gait, memory, vision, and etc. In other words, normal performance of even single function based on normal work of complex neuronal functional network (distributed in the central and peripheral nervous system) and providing efficient neuromuscular activation to sustain normal walking pattern or postural balance.

To recover or rehabilitate an affected function (for instance, like the gait), we must rehabilitate the whole functional network rather than separate parts of it (single muscle or muscular groups, local cortical or subcortical regions, etc.). Majority of neurological disorders lead to multiple damages (physical, cognitive, emotional, and mental) and cause a multidimensional package of symptoms. Multidimensional functional neurological damage needs multidimensional neurorehabilitation. Neurostimulation and neuromodulation approaches are contemporary tools for developing powerful, noninvasive, and integrated neurorehabilitation.

\subsection{Anatomical Targets}

Both emergent neurostimulation technologies cranial nerve non-invasive neuromodulation (CN-NINM), USA, 
and correction activity of the sympathetic nervous system (SYMPATOCORECTION), Russia, presented in current paper, are unique, original, and independently developed in different laboratories, but are surprisingly similar and complimentary to each other.

Both technologies are safe, noninvasive, subcortical, both use electrical pulse stimulation from battery driven device. Both methods are oriented to primary stimulation of CNS periphery, namely, the spinal or cranial nerves and corresponding neural ganglia.

In the SYMPATOCORECTION technology, the primary targets are complex of the neck spinal nerves (CICIV), neck truncus sympathicus, ganglion cervicalesuperius, ganglion ceruicale medium, cardiacuscervicalis inferior, vagus, stellate ganglion or plexus cervicalis.

The CN-NINM technology activates (through the lingual nerve (Vc) and chordatimpani (VII)) the corresponding ganglia geniculi and ganglion trigeminale. From here, the primary targets are several nuclei of the brainstem, namely, the trigeminal nuclei complex and solitary nuclei.

Both technologies create massive activation of adjacent parts of the CNS, namely, the neck segment of the spinal cord (SYMPATOCORECTION), or the brainstem and cerebellum (CN-NINM). In both cases, the authors observed noticeable positive effects in sensory and motor coordination, and changes in autonomic regulation, and both laboratories discovered the rehabilitation power of regularly applied stimulation.

\subsection{CN-NINMT Technology}

The portable neuromodulation stimulator (PoNS ${ }^{\mathrm{TM}}$ ) is an electrical pulse generator that delivers carefully-controlled electrical stimulation to the tongue. The pulses are generated and controlled by commercially available counter, timer, and wave-shaping electronic components. The components are mounted on a single printed circuit board (Figure 1).

The circuit board contains 143 gold-plated electrodes that contact the tongue. A rechargeable lithium-polymer battery with built-in charge safety circuitry provides the power.

The PoNS ${ }^{\mathrm{TM}}$ device is placed in the mouth and has been investigated in conjunction with physical therapy for treatment of balance and gait disorders caused by a variety of etiologies, including the traumatic brain injury, multiple sclerosis, central vestibular disorder, migraine-related balance disorder, chronic Meniere's disease, spinocerebellar ataxia, gentamicin ototoxicity, idiopathic cerebellar ataxia, peripheral vestibular disorder, idiopathic vestibular disorder, and cerebellar infarction [3]-[6].

The device is easily held at the place by the lips and teeth around the neck of the tab that goes into the mouth and rests on the anterior superior part of the tongue. The paddle-shaped tab of the device has a hexagonally patterned array of 143 gold-plated circular electrodes (1.50 mm diameter, on $2.34 \mathrm{~mm}$ centers). The array is created by a photolithographic process used to make printed circuit boards. It uses low-level electrical current to stimulate the lingual branch projections of at least two cranial nerves in the tongue anterior through the gold-plated elec-

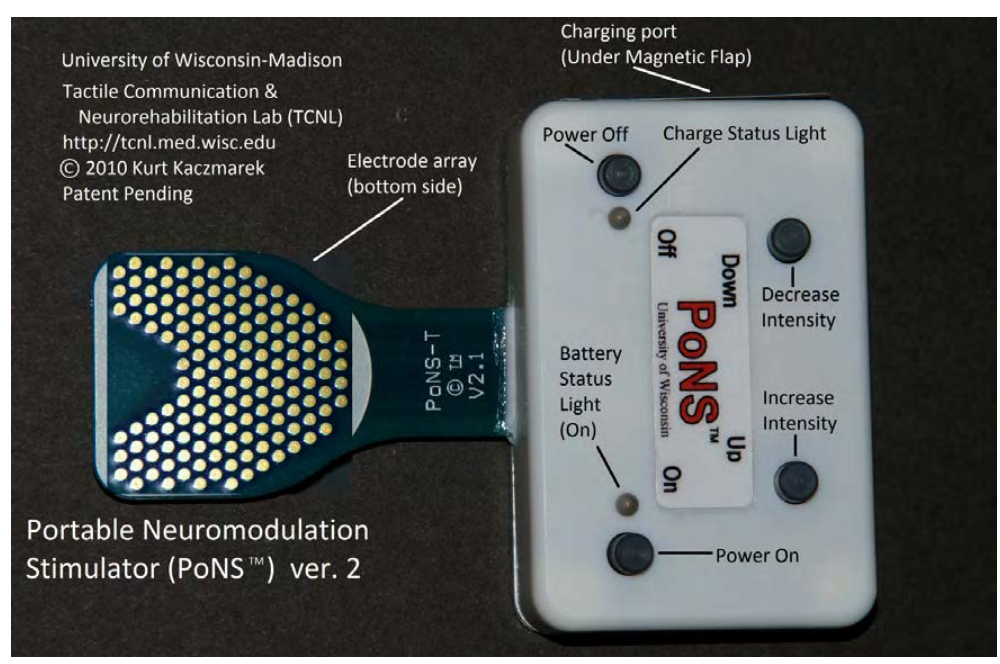

Figure 1. General view of the PoNS ${ }^{\mathrm{TM}}$ unit. 
trodes. Device function is user-controlled by four buttons: On, Off, Intensity “Up”, and Intensity "Down”. The system delivers triplets of 0.4 - $60 \mu$ s wide pulses at 5 ms intervals (i.e., of $200 \mathrm{~Hz}$ ) every $20 \mathrm{~ms}(50 \mathrm{~Hz})$ that has been designed to achieve a balance of stimulus dynamic range and sensation quality. The sensation produced by the array is similar to the feeling of drinking a carbonated beverage. The system has operational limits of $19 \mathrm{~V}$ (max) on the tongue (a nominal $5-7 \mathrm{kOhm}$ load). The biphasic waveform is specifically designed to ensure zero net DC current to minimize the potential for the tissue irritation.

The current hypothesis for the underlying mechanism by which the PoNS ${ }^{\mathrm{TM}}$ Cranial Nerve Noninvasive Neuromodulation (CN-NINM) stimulation leads to sustained neuromodulation (and subsequent therapeutic effect) comes from previous FMRI studies using optokinetic visual stimulation to activate regions involved in processing balance information [4]. Cortical procession of the motion is performed primarily by the motion sensitive visual cortex (hMT+). If these cortical regions determine that the visual data might contain information pertinent to maintaining balance, other brain regions including the parietal-insular vestibular cortex (PIVC), the vestibular nuclei, and cerebellar structures may be recruited to integrate the information with other sensory input.

Previous studies have shown that hMT+ is hypersensitive to visual motion in individuals with balance impairment. Previous work from our group investigating network behavior after the CN-NINM (Cranial Nerve Noninvasive Neuromodulation) showed hypersensitivity of the balance-processing network in individuals with balance dysfunction compared to healthy controls [4]. The network behavior normalized after the CN-NINM. A highresolution study of activity within the brainstem suggested that the trigeminal nucleus, the point at which afferents from the tongue enter the central nervous system, had altered neural responses to motion in the visual field after stimulation.

We hypothesize that spatio-temporal trains of spikes induced in the trigeminal and facial nerves by electrical stimulation of the tongue produce changes of activity in corresponding nuclei of the brainstem, namely, at least in the sensory and spinal nuclei of trigeminal nuclei complex (the largest nuclei in the brainstem, extending from the midbrain to the nuclei of the descending spinal tracts), and in the nucleus tractussolitarius where both stimulated nerves have direct projections. We postulate that intensive activation of these structures initiates a sequential cascade of changes in neighboring and/or connected nuclei by direct collateral connections, interneuron circuitry, or passive transmission of biochemical compounds in the intercellular space. Accordingly, electrotactile stimulation of cranial nerve endings, particularly in the lingual tract of the trigeminal and the facial nerve, initiate activity in the corresponding nuclei similar to long-term potentiation/inhibition, which, in the turn, increases the receptivity of multiple neural circuitries and/or affect internal mechanisms of homeostatic regulation.

This, in the turn, causes radiating therapeutic neurochemical and neurophysiological changes affecting both synaptic and extrasynaptic circuitries affecting information processing of afferent and efferent neural signals involved in the motion control including the cerebellum and nuclei of spinal motor pathways. PET scan study on the blind subjects before and after training with visual sensory substitution system via the tongue stimulation (electrotactile feedback) system demonstrates massive activation in cortical and subcortical levels of the brain [7].

It may also increase the receptivity of multiple neural circuitries and/or affect internal mechanisms of homeostatic regulation according to the contemporary concept of synaptic plasticity. We also cannot exclude also that this induces simultaneous activation of serotoninergic and noradrenalinergic regulation systems of the brain as well.

The result of this intervention is essentially brain plasticity on demand, i.e., a priming or up-regulating of targeted neural structures to develop new functional pathways that is the goal of neurorehabilitation and a primary means of functional recovery from permanent physical damage caused by a stroke or trauma. In the course of testing and training numerous persons having a primary indication of balance and gait disorder using the balance recovery therapeutic method we developed, we observed therapeutic benefits well beyond balance (attention, memory, multitasking, vision, fine motor control, sleep, tremor, tinnitus) regardless of their etiology (peripheral vestibular, central or idiopathic vestibular loss, cerebellar stroke, Meniere's, Parkinson's, MS). We believe on the theoretical grounds that our electrotactileneurostimulation activates and synchronizes several large brainstem nuclei and thecerebellum enabling the beneficial neuroplastic changes in multiple motor control circuitries. We envision the application of this principle to functional recovery of a broad range of sensory, motor, cognitive, and mood disorders caused by traumatic brain injury (TBI), stroke, concussions, and neurodegenerative diseases.

PoNS device was classified by FDA as Class 2 medical device, considered as non-significant risk technology 
and currently labeled as "For experimental use only". Since 2007, this emergent technology was tested in several case, pilot and control studies in US, for balance and gait rehabilitation in multiple sclerosis, stroke, Parkinson's disease and traumatic brain and spinal cord injury. Results of these studies were presented on several meetingsand conferences, several publications are now in press. Results of balance rehabilitation study and effects of brain activation were verified using fMRI technology [3] [6].

Control blind and double blind studies are on progress. Current update on our clinical research and development, experimental results and video materials can be accessed through website: tcnl.bme.wisc.edu.

\subsection{SYMPATOCORECTION Technology}

It is known that the following regulation contours participate in control of functional processes in the brain tissues: neurogenic, humoral, metabolic, and myogenic ones. These contours work in dynamic interaction. Their activity is targeted to providing physical homeostasis determined by the balance of water filtration from the blood into the brain tissue under influence of the hydrostatic pressure in the arterial part of capillary and water absorption in its venous part. This filtration is stipulated by the oncotic blood pressure and the chemical homeostasis of the brain internal environment [8]. Note that operation of any contour (mentioned above) maintains under presence of disturbances in limits of its physiological activity. We suggested using the transcutaneous electrical nerve stimulation (TENS) of the autonomic nervous system by the space-distributed field of low frequency current impulses (FCI). We apply the FCI to the neck nerve-knots projection of the sympathetic part of the autonomic nervous system. The FCI signals differ from typically used in the TENS by spatio-temporal pattern of stimulation, which are formed between the electrodes. In cathodes area, the current structure consists of spatially distributed partial impulses. The impulse duration ranges from 30 to $50 \mu \mathrm{sec}$ that is correspond to velocity of creating the excitation in the myelinatednerve fibers. In the anodes area, the impulse structure consists of spatially concentrated structure of the partial impulses. Number of impulses in the structure is equal to the number of cathodes, and the impulse duration ranges from 400 to $600 \mu$ sec that is correspond to velocity of creating the excitation in the nonmyelinated nerve fibers [9]. The scheme for forming the FCI is shown in Figure 2.

The neurogenic component of regulation of the brain blood circulation is mainly implemented by variation of the vascular tonus of the large brain arteries and arteries inserted onto the brain tissue. This component is determined by tonic activity of the sympathetic adrenergic vasoconstrictive nerve fibers coming out the neck nerve-

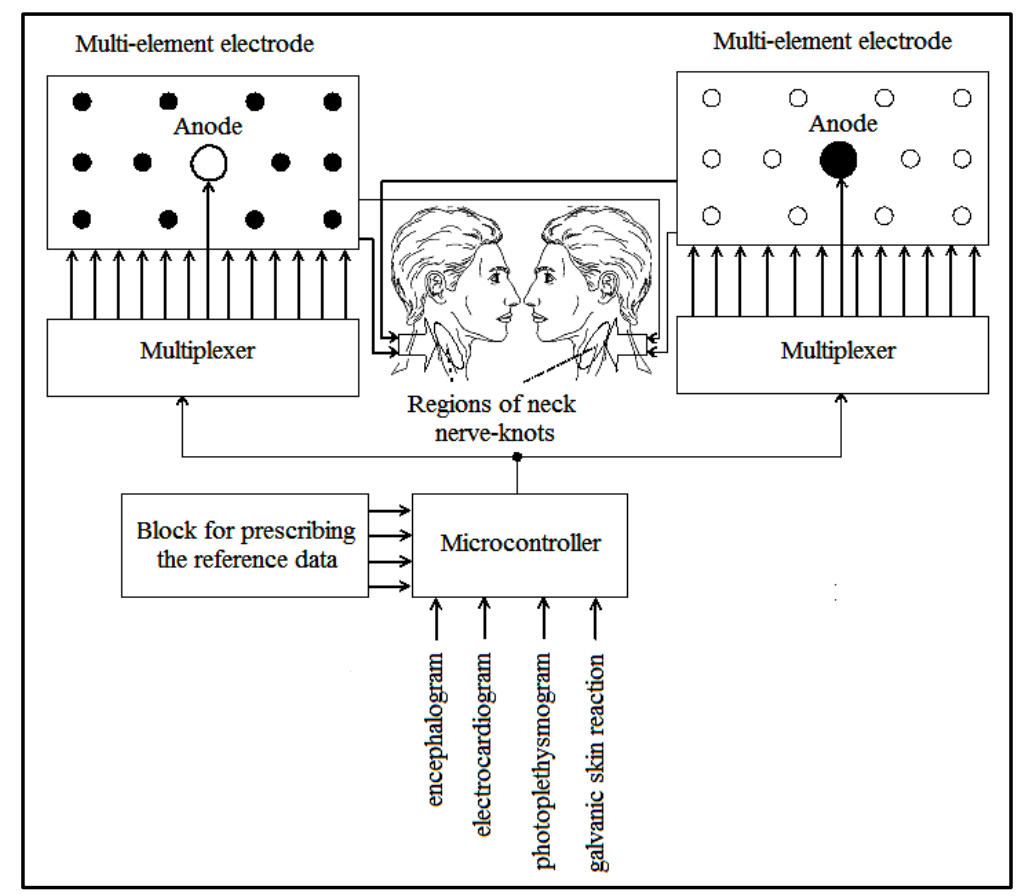

Figure 2. The scheme for forming the FCI. 
knots. Increasing of the tonus led to increasing the resistance to the blood flow, and reduction of the tonus leads to decreasing the resistance. The effectiveness of such regulation is weakened with decreasing the vascular diameter. The main method of the neural regulation of the microcirculation can be in nonsynaptic innervation with a free diffusion of neurotransmitters in the direction of blood vessels. Indeed, it seems to be true, because nonsynaptic regulation of the whole vaso-microlocus (but not separate capillaries) is crucial for forming the microcirculation blood flow.

SYMPATOCORRECTION methodology is based on ability to control the dynamic correction activity of the sympathetic nervous system (DCASNS). It is also known, that practically in any dynamic multi-contour stabilization system, variation of a process in one loop of control causes a compensatory variation in other loops.

The similar changes can occur in the regulatory circuits providing stability of the main characteristics of homeostasis. To achieve it, the special functional system was formed in a human body. The main organizing factor of operation of such a system is satisfaction of the organism current needs. The core architectonics of such functional systems should include the following main sequential processing stages: afferent synthesis, decision solution, acceptor of the action result, efferent synthesis, and evaluation of the final result, achieved by the feedback afferentation.

The afferent synthesis stage is the initial one. During of this process, is performed the synthesis of excitations caused by internal metabolic demand, situational and starting afferentation using individually acquired genetic mechanisms memory continuous. The afferent synthesis stage is lead to the decision making stage. In its physiological essence, this stage means restriction of degrees of freedom for functional system operation and selection of the unique scenario of the effector actions, targeted on satisfaction of the main body need that was defined on the afferent synthesis stage [10].

To modulate DCASNS regulation processes, we should have ability to manipulate the conductivity of the neck sympathetic stem by means of the FCI. Inhibition or facilitation of its activity causes the neurogenic and myogenic regulation of cerebral blood flow. This is due to the constrictive and humoral affection onto the tonus of the brain vessels of various calibers: form the main arteries to the capillaries. This effect is confirmed by results of an experimental simulation on laboratory rats [11] [12], radio-isotopic investigations on patients-volunteers [13] and, also, by clinic results of application of the "SYMPATOCOR-01" unit [14]. Its general view is presented in Figure 3.

SYMPATOCOR was originally registered in Russian Federation in year 2000. Device was certified according to state standard medical devices of Russian Federation.

\subsection{Preliminary Results}

Application of the "SIMPATOCOR-01" device is certified on territory of the Russian Federation by the State Inspection for health service and social development.

Depending on pathogenesis of functional violations of the head brain the sympathetic correction, algorithm has structural organization of two types:

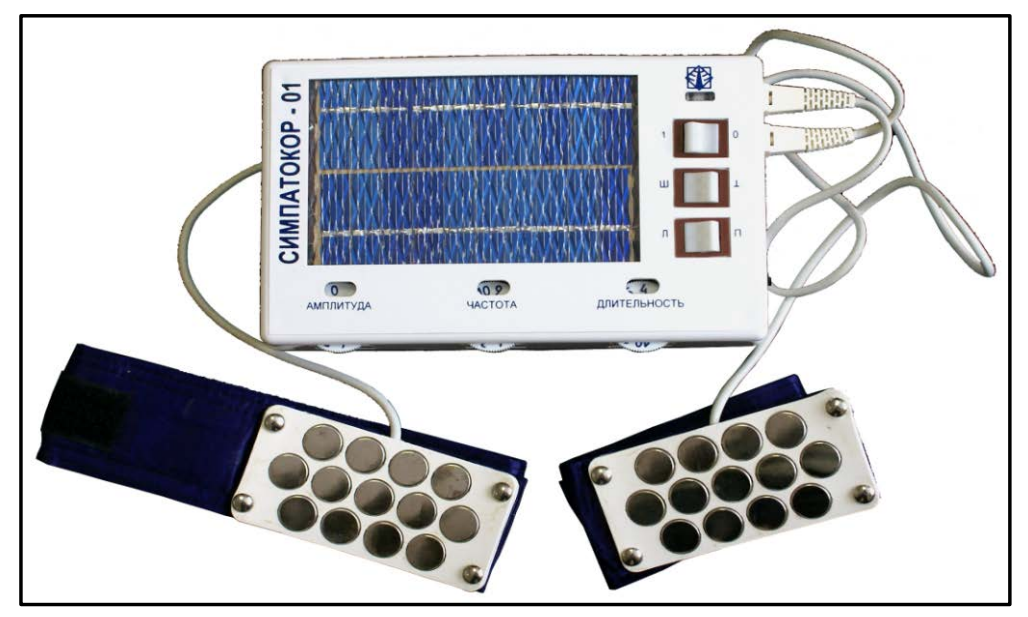

Figure 3. General view of the "SYMPATOCOR-01" unit. 
- In case of the peripheral functional abnormalities, the major goal is the normalization of autonomic nervous system balance: the appropriate state of balance is determined by the ratio of activities of the sympathetic and parasympathetic parts of this system;

- In case of the functional central brain abnormalities, the goal of normalization is control is the optimization of the bioelectric activity brain (EEG rhythms).

Currently, the "SIMPATOCOR-01" device is used in more than 200 Russian hospitals. It is applied to correct and control the following pathologies: migraine, neurocirculatory dystonia, traumatic brain injury and brain concussion, alcohol and narcotic abstinences, hypertonic diseases, obliterate atherosclerosis of the lower limbs, Raynaud's disease, trigeminal nerve inflammation, sensorineural deafness, degenerative diseases of vision and atrophy of the optical nerve, osteochondrosis of the back bone, neuropathies of various genesis, cephalalgia syndrome, hyperhidrosis syndrome, syndrome of the orthostatic hyposthenia and postural tachycardia, vestibular disease, vegetative deregulation syndrome, epilepsy.

Also, the devices is used for rehabilitation of stroke, physiotherapy in gynecology of adolescents, for effective substitution of invasive blockades, in curing the depression, tics (facial, mesencephalic, speech, psychic), attention deficit hyperactivity disorder, and etc. [15].

\section{Conclusions}

The CN-NINM technology is oriented to stimulate the multiple brain structures (starting from brainstem and cerebellum), including regions of the sensory analysis and centers of the motion control.

The SYMPATOCORECTION system stimulates the parts of the sympathetic nervous system that participate in organization of the brain and body circulatory system, largely affecting autonomic neural system and also directly or indirectly can affect sensory or motor systems. In this way, the SYMPATOCORECTION provides complimentary and partially overlapped neurostimulation pattern.

We suppose that combined simulation of different functional systems in the brain can significantly increase effectiveness of functional processes, and improve control of the brain recovery and rehabilitation.

It can be achieved by the appropriate use of CN-NINM and SYMPATOCORECTION systems. The research of effectiveness and efficacy of simultaneous application both CN-NINM and SYMPATOCORECTION is an intriguing and attractive discovery that needs extensive and intensive further researches.

We should emphasize that both technologies may be applied as a standalone therapy, but we envision that to achieve maximal neurorehabilitation, they would be applied in conjunction with existing rehabilitation or recovery training procedures.

Ideally, we envision this practical and portable application of our technologies as an inexpensive and easy-touse "neurorehabilitation toolbox", applicable in any stationary or field hospital or other medical facility.

Both technologies were developed to use in a home-therapy setting. The appeal here is that we can capitalize on the innate motivation of neurological patients to return to full function, so that therapeutic training can be established in the clinic, but the majority of the actual rehabilitation can be done at the place, rate and ability of the individual. With periodic retraining intervals, the application of non-invasive stimulation outside of the actual clinical setting has the potential to greatly amplify and accelerate the effects of an advanced therapeutic regime.

\section{Acknowledgements}

The authors take this opportunity to gratefully acknowledge the assistance and contributions of the of the few collaborators: Spector S., Azin A., Stecher O., Korotkikh S., Shershever A., Retyunsky K., Abdulkerimov H., Danilova I., Badtieva V., Kazakov J., Petrenko T., who helped to develop and test SYMPATOCORECTION technology in Ekaterinburg, Russia. Deserving of special mention are M. Tyler and K. Kaczmareck, who create the electorotactile stimulation system and developed several generation of the technology, including CN-NINM in Madison, USA.

\section{References}

[1] Weaver, F.M., et al. (2009) Bilateral Deep Brain Stimulation vs. Best Medical Therapy for Patients with Advanced Parkinson Disease: A Randomized Controlled Trial. JAMA, 301, 63-73. http://dx.doi.org/10.1001/jama.2008.929

[2] Saiote, C., et al. (2013) Combining Functional Magnetic Resonance Imaging with Transcranial Electrical Stimulation. Frontiers in Human Neuroscience, 7, 435. http://dx.doi.org/10.3389/fnhum.2013.00435 
[3] Wildenberg, J.C., et al. (2013) Altered Connectivity of the Balance Processing Network after Tongue Stimulation in Balance-Impaired Individuals. Brain Connect, 3, 87-97. http://dx.doi.org/10.1089/brain.2012.0123

[4] Wildenberg, J.C., et al. (2011) High-Resolution fMRI Detects Neuromodulation of Individual Brainstem Nuclei by Electrical Tongue Stimulation in Balance-Impaired Individuals. Neuroimage, 56, 2129-2137. http://dx.doi.org/10.1016/j.neuroimage.2011.03.074

[5] Wildenberg, J.C., et al. (2011) Electrical Tongue Stimulation Normalizes Activity within the Motion-Sensitive Brain Network in Balance-Impaired Subjects as Revealed by Group Independent Component Analysis. Brain Connect, 1, 255-265. http://dx.doi.org/10.1089/brain.2011.0029

[6] Wildenberg, J.C., et al. (2010) Sustained Cortical and Subcortical Neuromodulation Induced by Electrical Tongue Stimulation. Brain Imaging Behaviour, 4, 199-211. http://dx.doi.org/10.1007/s11682-010-9099-7

[7] Ptito, M. and Kupers, R. (2005) Cross-Modal Plasticity in Early Blindness. Journal of Integrative Neuroscience, 4, 479-488. http://dx.doi.org/10.1142/S0219635205000951

[8] Moskalenko, J.E. (1980) Biophysical Aspects of Cerebral Circulation. Pergamon Press, Oxford, New York.

[9] Kublanov, V.S. (1998) Electrophysics Method of Performing a Procedure to Correct Violations of the Regulation of Cerebral Blood Flow. Biomedical Radioelectronics, 3, 42-49.

[10] Chernih, A.M., Alexandrov, P.N. and Alekseev, O.V. (1984) Microcirculation. Medicine, Moskow.

[11] Kublanov, V.S., Danilova, I.G., Goette, I.F., et al. (2010) Application of Spatially Distributed Field of Electric Impulses for Correction of Angiogenesis in Ischemic Muscular Tissue. International Journal of Biomedical Science, 6, 310315.

[12] Kublanov, V.S., Danilova, I.G., Goette, I.F., et al. (2012) Experimental Simulation of the Effects of Autonomic Regulation for the Correction of Immobilization Stress Rats. Biomedical Radioelectronics, 8, 56-67.

[13] Kublanov, V.S., Shershever, A.S., Lavrova, C.A., et al. (2004) Treatment of Epilepsy Using Spatially Rotating Fields of the Current Pulses. Biomedical Radioelectronics, 5-6, 4-15.

[14] Kazakov, J.E., Retyunsky, K.Ju., Kublanov, V.S, et al. (2011) The Experience of Using “SIMPATOCOR-01” Apparatus for the Correction of the Symptoms of Anxiety and Depression in Patients with Hypertonic Diseases. Post-Traumatic and Post-Stress Conditions in Medicine: The Possibility of Rehabilitation, Ekaterinburg, CB-96, 86-87.

[15] Kublanov, V.S., Shmirev, V.I., Shershever, A.S., Kazakov, J.E., et al. (2010) About Innovative Possibilities of Device "SIMPATOCOR-01" in Management of Functional Disorders of Vegetative and Central Nervous System in Neurology, Kremljovskaya Medicine. Clinichesky Vestnik, 4, 60-64. 